\title{
Does the world need germline editing for $\beta$-thalassemia?
}

Andreas E. Kulozik

Chairman, Department of Pediatric Oncology, Hematology and Immunology, University of Heidelberg, Hopp-Children Cancer Center Heidelberg (KiTZ), Germany

E-mail: andreas.kulozik@med.uni-heidelberg.de

https://doi.org/10.3324/haematol.2021.279998

In this issue of Haematologica, Lu and co-workers report the correction, by CRIPSR-Cas 9 gene editing, of the $\beta$-globin gene in the germ cells of a mouse model carrying the human $\beta$-globin gene with the IVS2-654 thalassemia $\left(\beta^{654}\right)$ mutation that is common in East Asian patients with $\beta$ thalassemia. This mutation creates a new donor splice site in intron 2 of the $\beta$-globin gene which co-operates with a cryptic splice site further downstream to insert an abnormal exon containing a premature stop codon. This mutation thus results in the almost complete inactivation of the affected $\beta$-globin allele. Lu and co-workers designed two sgRNA that simultaneously target and delete both the novel 5' donor splice site and the cryptic acceptor splice site. Microinjection of the two sgRNA into fertilized murine eggs together with the nuclease Cas 9 produced $12 / 37$ viable mice with editing of the target locus, half of which carried the desired deletion of the target region. Remarkably, the peripheral blood of seven of the 12 edited mice showed correct splicing of the $\beta$-globin gene, and six of these seven exclusively expressed correctly processed RNA and normal human globin. Finally, the authors demonstrated that mice with successfully edited $\beta^{654}$ human $\beta$-globin genes have much-improved hematologic parameters and survival compared to nonedited mice. ${ }^{1}$ These results indicate that the faulty RNA processing induced by the common $\beta^{654}$ thalassemia mutation can be corrected by a complex and innovative editing strategy.

$\beta$-thalassemia is one of the most common genetic disorders worldwide and has been a target for the development of gene therapy for decades. In fact, the $\beta$-globin gene was the first human gene to be cloned more than 40 years ago and more than 300 mutations resulting in thalassemia have been described since then. Early attempts at gene therapy were not successful. ${ }^{2}$ However, with the advent of modern vector technology the first reports of successful somatic thalassemia gene therapy of hematopoietic cells began to emerge some 10 years ago. ${ }^{3}$ More recently, systematic clinical studies have employed self-inactivating lentiviral vectors containing a therapeutic $\beta$-globin gene. Such constructs are used to transduce human hematopoietic stem cells mobilized and isolated from affected patients thus adding a functional $\beta$-globin gene into the genome of these cells playing a key role in the pathogenesis in thalassemia. ${ }^{4}$ The game-changing efficacy and safety of this procedure convinced the European Medicine Agency (EMA) to license such a product for the treatment of a defined group of patients with transfusion dependent $\beta$-thalassemia.

Reversing the perinatal hemoglobin switch from fetal to adult globin synthesis by CRIPSR-Cas9-mediated inactivation of BCL11A, the central erythroid-specific negative regulator of $\gamma$-globin gene expression, in hematopoietic stem cells has been reported to induce high-level HbF synthesis resulting in transfusion independence. ${ }^{5}$ However, both gene addition and gene editing strategies may potentially carry the risk of serious long-term complications by insertional mutagenesis. ${ }^{6}$ Concerns about the safety of gene editing have recently been raised by reports describing that the double-stranded DNA breaks induced by Cas9 can trigger a TP53-mediated DNA damage response and major structural changes of the DNA resulting in the formation of micronuclei and chromothripsis, one of the major mechanisms of carcinogenesis. ${ }^{7,8}$ While these safety concerns have so far not been an issue in either gene addition or gene editing studies in patients with $\beta$ thalassemia, long-term follow-up will inform us whether such concerns will be relevant in the long run.

When considering the therapeutic use of genetic engineering it is conceptually important to distinguish between somatic gene therapy targeting a disease-relevant cell type or tissue and germline engineering that introduces heritable genetic changes. While the former strategy has been used to develop novel treatments of several genetic and acquired diseases, the latter is commonly used in animal models aimed at the understanding of key pathological mechanisms. In fact, manipulation to introduce heritable changes into human germ lines has been viewed very critically by several European, NorthAmerican and Chinese scientific societies and is legally banned in the European Union. .,10 $^{9}$

Despite this, Lu and co-workers consider that the results of their manipulation of murine germ cells "provide a groundwork for the exploration of $\beta^{654}$-thalassemia therapy in the future". Notably, these authors report that the manipulation of fertilized murine eggs induced several unexpected structural variants including inversions, unexpected single nucleotide substitutions and larger deletions than those the pair of SgRNA were designed to generate. These findings thus highlight the potential of gene editing to induce unexpected genetic variants that go beyond simple off-target effects induced by sequence similarities between the guide RNA and other loci of the genome. Consistent with the findings of Pellman's group in edited 
hematopoietic stem cells ${ }^{7}$ these findings indicate that gene editing is more imprecise than widely thought and that its clinical use should be developed with caution. While this is certainly true for the development of somatic gene therapy, these findings are clearly particularly relevant when heritable manipulation of human germ cells is considered for the exploration of $\beta$-thalassemia therapy. Even with a perfectly precise technology the ethical concerns of introducing heritable changes into human germ cells have been grave enough to ban human germline engineering for therapeutic purposes. And to those who do not share the concept of ethical reservations against germline manipulation, the substantial technical uncertainties relating to the lack of specificity of the procedure should be reason enough to stand off. One might therefore wonder whether Lu and coworkers may want to adapt their innovative technical approach to the development of somatic gene therapy, which may also carry the potential of risk but does not cause heritable changes of germ cells thus limiting any potential unwanted outcome to the individual patient.

\section{Disclosures}

AEK has received honoraria from Novartis, Sanofi, Novo Nordisk, Celgene and Bluebird Bio.

\section{References}

1. Lu D, Gong X, Fang Y, et al. Correction of RNA splicing defect in B654-thalassemia mice using CRISPR/Cas9 gene-editing technology. Haematologica. 2022;107(6)1427-1437.

2. Kolata GB, Wade N. Human gene treatment stirs new debate. Science. 1980;210(4468):407.

3. Cavazzana-Calvo M, Payen E, Negre O, et al. Transfusion independence and HMGA2 activation after gene therapy of human $\beta$-thalassaemia. Nature. 2010;467(7313):318-322.

4. Thompson AA, Walters MC, Kwiatkowski J, et al. Gene therapy in patients with transfusion-dependent $\beta$-thalassemia. N Engl $J$ Med. 2018;378(16):1479-1493.

5. Frangoul H, Altshuler D, Cappellini MD, et al. CRISPR-Cas9 gene editing for sickle cell disease and $\beta$-thalassemia. N Engl J Med. 2021;384(3):252-260.
6. Kunz JB, Kulozik AE. Gene therapy of the hemoglobinopathies. Hemasphere. 2020;4(5):e479.

7. Leibowitz ML, Papathanasiou S, Doerfler PA, et al. Chromothripsis as an on-target consequence of CRISPR-Cas9 genome editing. Nat Genet. 2021;53(6):895-905.

8. Haapaniemi E, Botla S, Persson J, Schmierer B, Taipale J. CRISPR-Cas9 genome editing induces a p53-mediated DNA damage response. Nat Med. 2018;24(7):927-930.

9. Coller BS. Ethics of human genome editing. Annu Rev Med. 2019;70:289-305.

10. Büning $H$, Griesenbach $U$, Fehse B, et al. Consensus statement of European societies of gene and cell therapy on the reported birth of genome-edited babies in China. Hum Gene Ther. 2018;29(12):1337-1338. 